\title{
$K$-THEORY OF AZUMAYA ALGEBRAS
}

\author{
CHARLES A. WEIBEL
}

\begin{abstract}
Quillen has defined a $K$-theory for symmetric monoidal categories. We show that Quillen's groups agree with the groups $K_{0}, K_{1}$, and $K_{2}$ defined by Bass. Finally, we compute the $K$-theory of the Azumaya algebras over a commutative ring.
\end{abstract}

The purpose of this paper is to advertise the $K$-theory of symmetric monoidal categories, and to compute the $K$-theory of the category of Azumaya $R$-algebras. The point is that Quillen's theory (introduced in [6]) is a natural generalization of the "classical" theory for $K_{0}, K_{1}, K_{2}$ defined by Bass in [2], [3], [4]. On the other hand, it provides a wealth of examples of infinite loop spaces (see [1], [9], [10], [12] and [13]).

A symmetric monoidal category is a category $S$ with a unit $0: * \rightarrow S$ and a product $\square: S \times S \rightarrow S$ which is commutative and associative up to coherent natural isomorphism; the precise definition may be found in [7]. We shall be especially interested in the following examples (from [2]):

(1) $\mathbf{P}$, the fin. gen. projective modules over a ring $R$. The product $\square$ is direct sum, and we consider only isomorphisms.

(2) FP, the fin. gen. faithful projective modules over a commutative ring $R$. The product $\square$ is the tensor product, and the arrows are isomorphisms.

(3) Pic, the full subcategory of FP of rank one projective modules.

(4) Az, the Azumaya algebras over a commutative ring $R$. The arrows are $\boldsymbol{R}$-algebra isomorphisms, and the product is the tensor product. If $R$ is a field an Azumaya algebra is just a central simple algebra.

In the language of [3, Chapter VII], a symmetric monoidal category is a "category with product $\square$ ", with the additional condition that there be a special object 0 and natural isomorphisms $0 \square s \cong s \cong s \square 0$ satisfying the coherence conditions on page 159 of [7]. Groups $K_{i}^{\operatorname{det}}(S)(i=0,1,2)$ were defined and studied in [2], [3] and [4], using only the objects, isomorphisms and product of the category $S$.

We will restrict our attention to the category SMCat of small symmetric monoidal categories and relaxed morphisms. We require in addition that every symmetric monoidal category $S$ in $S M C a t$ satisfies (i) every arrow is an isomorphism, and (ii) every translation $s \square: \operatorname{Aut}(t) \rightarrow \operatorname{Aut}(s \square t)$ is an injection. The categories P, FP, Pic, Az all belong to SMCat, as do the categories:

Received by the editors November 29, 1979.

AMS (MOS) subject classifications (1970). Primary 18F25; Secondary 18D10, 16A16, 55P47.

Key words and phrases. Algebraic $K$-theory, Azumaya algebra, infinite loop space, symmetric monoidal category.

${ }^{1}$ Supported by NSF grant MCS-79-03537.

() 1981 American Mathematical Society $0002-9939 / 81 / 0000-0001 / \$ 02.75$ 
(5) $\operatorname{Quad}^{\lambda}(A, \Lambda)$, the category of nonsingular $(\lambda, \Lambda)$-quadratic $A$-modules defined in [4]. Here $A$ is a ring with involution, $\lambda$ is a central element of $A$ satisfying $\lambda \bar{\lambda}=1$, and $\Lambda$ is a additive subgroup of $\{a \in A: a=-\lambda \bar{a}\}$ containing $\{a-\lambda \bar{a}\}$ and closed under $r \mapsto a r \bar{a}$. The product is direct sum. The principal goal of [4] was to calculate the groups $K_{1}^{\operatorname{det}}\left(Q_{u_{a d}}{ }^{\lambda}\right)$ for various $(A, \lambda, \Lambda)$.

(6) Ens, the category of finite sets and their isomorphisms, the product being disjoint union. It is easy to see that $K_{0}^{\text {det }}(\mathbf{E n s})=\mathbf{Z}, K_{1}^{\text {det }}($ Ens $)=\{ \pm 1\}$; it is known (see [1]) that the Quillen $K$-groups $K_{i}$ (Ens) are the "stable stems" $\pi_{i}^{s}$ $=$ colim $\pi_{n+i}\left(S^{n}\right)$. The "free module" functor from Ens to $\mathbf{P}(\mathbf{Z})$ induces the map $\pi_{*}^{s} \rightarrow K_{*}(\mathrm{Z})$.

1. Quillen $K$-theory. In [6], Quillen defined groups $K_{*}(S)$ for every $S$ in $S M C a t$. This is achieved by associating to every $S$ in $S M C a t$ a new symmetric monoidal category $S^{-1} S$ (not in $S M C a t$ ) properly containing $S$. Applying geometric realization yields a topological space $B S^{-1} S$; the groups $K_{*}(S)$ are defined to be the homotopy groups $\pi_{*}\left(B S^{-1} S\right)$. It is shown in [6] that the groups $K_{*}(P)$ coincide with the algebraic $K$-groups $K_{*}(R)$ of the underlying ring $R$.

One pleasing property of these topologically defined groups is that they agree with the classically defined $K$-groups. Classically, $K_{0}^{\text {det }}(S)$ is the group completion of the abelian monoid of isomorphism classes of objects of $S$. Bass (in [2], [3]) defined $K_{1}^{\text {det }}(S)$ to be the direct colimit of the groups $H_{1}\left(\operatorname{Aut}_{S}(s)\right)=$ $\operatorname{Aut}(s) /[\operatorname{Aut}(s), \operatorname{Aut}(s)]$.

Proposition 1. Quillen's groups $K_{i}(S)$ agree with Bass's groups $K_{i}^{\operatorname{det}}(S)$ for $i=0,1$.

Proof. From [6] we know that $H_{*}\left(B S^{-1} S\right)=$ colim $H_{*}(B S)$, where the colimit is taken over the directed set of (isomorphism classes of) objects $s$ in $S$ under translation. For $*=0$ we obtain the $K_{0}$ result. Reading this for $*=1$ yields $K_{1}(S)=\pi_{1}\left(B S^{-1} S\right)=H_{1}\left(B_{0} S^{-1} S\right)=\operatorname{colim} H_{1}(B \operatorname{Aut}(s))=K_{1}^{\operatorname{det}}(S)$.

Remark. In [4], Bass defined groups $K_{2}^{\text {det }}(S)$. In the next section we will show that this agrees with the $K_{2}(S)$ of Quillen.

Another pleasing property is that the spaces $B S^{-1} S$ are infinite loop spaces. This follows from the fact that $\pi_{0} B S^{-1} S$ is the group $K_{0}^{\text {det }}(S)$ and Proposition 2 below. For example, $B$ Ens ${ }^{-1}$ Ens is the space $\Omega^{\infty} \Sigma^{\infty}$, and $B \mathbf{P}^{-1} \mathbf{P}$ is the space $K_{0}(R) \times B$ $\mathrm{Gl}(R)^{+}$(see page 91 of [1]).

Proposition 2. If $T$ is a small monoidal category, BT is a homotopy associative $H$-space. If $T$ is symmetric monoidal, BT is also homotopy commutative, and BT is an infinite loop space if and only if $\pi_{0}(B T)$ is an abelian group.

REMARK. There is a simple, purely algebraic definition of $\pi_{0}(B T)$. If $T$ is a small symmetric monoidal category, define $\pi_{0} T$ to be the set of objects of $T$, modulo the equivalence relation generated by requiring $s \sim t$ whenever there is an arrow from $s$ to $t$. The product $\square$ makes $\pi_{0} T$ an abelian monoid. If $T$ is in $S M C a t, \pi_{0} T$ is the monoid of isomorphism classes of objects. Since $\pi_{0} T$ is $\pi_{0}(B T)$, the topological space $B T$ is an infinite loop space iff $\pi_{0} T$ is a group. 
Proof. The functor $\square: T \times T \rightarrow T$ induces $B \square: B T \times B T \cong B(T \times T) \rightarrow B T$, making $B T$ an $H$-space. Associativity (and commutativity in the symmetric case) of up to natural equivalence translates directly into homotopy associativity (and commutativity) of $B T$. To determine when $B T$ is an infinite loop space, we use Segal's machine [12]; this is appropriate since Thomason has shown in [13] that $B T$ is the initial space of a $\Gamma$-space. By [15, p. 461], $B \square$ has a homotopy inverse iff $\pi_{0}(B T)$ is a group, and by [12] this is necessary and sufficient for $B T$ to be an infinite loop space.

Remark. We could have also used May's machine. In the relevant vocabulary, $B T$ is an $A_{\infty}$ space if $T$ is monoidal, and $B T$ is an $E_{\infty}$ space if $T$ is symmetric monoidal. This was shown in [9]. The above formulation of Proposition 2 was shown to me by $Z$. Fiedorowicz.

The usefulness of Proposition 2 is that some of the $S$ in SMCat already have a group for $\pi_{0} S$. In this case, the natural map $B S \rightarrow B S^{-1} S$ is a homotopy equivalence (it is an infinite loop space map which is a homology isomorphism). For example, this is true of $S=$ Pic. It follows from [2] or [3] that $B$ Pic $\simeq \operatorname{Pic}(R) \times$ $B U(R)$, where $\operatorname{Pic}(R)$ is the Picard group of the commutative ring $R$, and $U(R)$ is the group of units of $R$. We have the

Corollary. $K_{0}$ Pic $=\operatorname{Pic}(R), K_{1}$ Pic $=U(R)$, and the groups $K_{*}$ Pic are zero for $* \geq 2$.

2. The plus construction and $K_{2}$. If the category $S$ has a countable, cofinal subcategory, we can construct a group $\operatorname{Aut}(S)$ playing the role that $\mathrm{Gl}(R)$ does for P. The construction is given on page 355 of [3], although the constructions of [2, p. 25], [4, p. 197], and [14] may be used where appropriate.

The groups $\operatorname{Aut}(S)$ are easy to compute in the sample categories given in the introduction. The free modules in $P$ and FP allow us to take $\operatorname{Aut}(\mathbf{P})=\operatorname{Gl}(R)$, $\operatorname{Aut}(\mathbf{F P})=\mathrm{Gl}_{\otimes}(R)=\operatorname{colim}\left\{\mathrm{Gl}_{n}(R) ; \alpha \mapsto \alpha \otimes I\right\}$. Aut(Pic) is just $U(R)$. The matrix rings in Az allow us to take Aut(Az) to be the direct colimit of the $R$-algebra automorphisms of the $M_{n}(R)$. We have $\operatorname{Aut}\left(\operatorname{Quad}^{\lambda}(A, \Lambda)\right)=U^{\lambda}(A, \Lambda)=$ colim $U_{2 n}^{\lambda}(A, \Lambda)$ and Aut(Ens) $=\Sigma_{\infty}=\operatorname{colim} \Sigma_{n}$.

Proposition 3. Suppose that $S$ has a countable, cofinal subcategory, so that $\operatorname{Aut}(S)$ exists. Then the commutator subgroup $E$ of $\operatorname{Aut}(S)$ is a perfect, normal subgroup, so the plus construction may be applied to $B \operatorname{Aut}(S)$. The resulting space is the basepoint component of $B S^{-1} S$, i.e., $B S^{-1} S \simeq K_{0}(S) \times B \operatorname{Aut}(S)^{+}$. Moreover, $K_{1}(S)=\operatorname{Aut}(S) / E$.

Proof. As $E$ is a direct colimit, every element of $E$ is a product of elements, each represented by a commutator $[\alpha, \beta]$ in some Aut $(s)$. We compute in $\operatorname{Aut}(s \square s \square s)$ that $[\alpha, \beta] \square 1 \square 1=\left[\alpha \square \alpha^{-1} \square 1, \beta \square 1 \square \beta^{-1}\right]$, which represents an element of $[E, E]$ by the Abstract Whitehead Lemma on page 351 of [3]. This shows that $E$ is perfect, so that $f: B \operatorname{Aut}(S) \rightarrow B \operatorname{Aut}(S)^{+}$exists and is any acyclic map with $\operatorname{ker}\left(\pi_{1} f\right)=E$. If we copy the telescope construction of [6], we obtain such an acyclic map from $B \operatorname{Aut}(S)$ to the basepoint component of $B S^{-1} S$, proving the proposition. 
We are now in a position to compare Quillen's $K_{2}$ to Bass's $K_{2}^{\text {det }}$. In Appendix A to [4], Bass defined $K_{2}^{\text {det }}(S)$ to be the direct colimit of the groups $H_{0}(\operatorname{Aut}(s) ;[\operatorname{Aut}(s), \operatorname{Aut}(s)])$.

We remark that when $\operatorname{Aut}(S)$ exists we have $K_{2}^{\text {det }}(S)=H_{2}(E)$. This may be seen by reading the proof of (A.6) on page 200 of [4]. In this case, $K_{2}^{\text {det }}(S)$ may also be interpreted as the kernel of a universal central extension of the perfect group $E$ (as in [11]).

THEOREM 4. Quillen's $K_{2}(S)$ is the same as Bass's $K_{2}^{\operatorname{det}}(S)$.

Proof. Any $S$ in SMCat is the direct colimit of full subcategories which are countable, and hence for which $\operatorname{Aut}(S)$ exists. As Bass's and Quillen's groups both commute with direct colimits, we are reduced to proving the theorem when $\operatorname{Aut}(S)$ exists. In this case we have to show that $K_{2}(S)=H_{2}(E)$. We will use a modification of the proof of Proposition 4.12 in [9], which is essentially due to D. W. Anderson.

There is a homotopy fibration $B E \rightarrow B \operatorname{Aut}(S) \rightarrow B\left(K_{1} S\right)$. Since $K_{1} S$ is an abelian group, $B\left(K_{1} S\right)$ is an Eilenberg-Mac Lane space. The map $B \operatorname{Aut}(S) \rightarrow$ $B\left(K_{1} S\right)$ factors through an $H$-space map $B \operatorname{Aut}(S)^{+} \rightarrow B\left(K_{1} S\right)$ by universality of the plus construction. If $F$ denotes the fiber of the latter map, there is a map of fibrations:

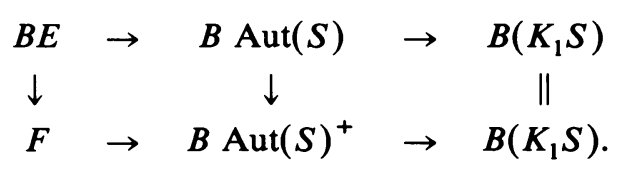

The action of $K_{1} S=\pi_{1} B\left(K_{1} S\right)$ on $B E$ is trivial for the same reasons given in [9]: if $y \in \operatorname{Aut}(s)$ represents $[y] \in K_{1} S$ and $z \in H_{*}(B E)$, we can choose a subgroup $\operatorname{Aut}(t)$ of $\operatorname{Aut}(s \square t)$ for some $t$ so that $z$ is in the image of $H_{*}(B[\operatorname{Aut}(t), \operatorname{Aut}(t)])$. As $y$ commutes with Aut $(t),[y]$ acts trivially on $z$. On the other hand, the action of $K_{1} S$ on $H_{*}(F)$ is trivial because $F$ is connected and is the fiber of an $H$-space map (see [5, p. 16-09]). It follows by the Comparison Theorem (in [8]) that $H_{*}(E)=$ $H_{*}(B E) \rightarrow H_{*}(F)$ is a homology isomorphism. On the other hand, $F$ is simply connected, so $H_{2}(F) \cong \pi_{2}(F) \cong \pi_{2}\left(B \operatorname{Aut}(S)^{+}\right)=K_{2}(S)$.

We will need the following result which is implicit in [10, p. 96], and was pointed out in [14]. The proof involves a comparison of the groups $\mathrm{Gl}(R)$ and $\mathrm{Gl}_{\otimes}(R)$.

Proposition 5. $K_{*}(\mathrm{FP})=\mathbf{Q} \otimes K_{*}(\mathbf{P})=\mathbf{Q} \otimes K_{*}(R)$ for $*>1$, while $K_{0}(\mathbf{F P})=$ $U^{+}\left(\mathbf{Q} \otimes K_{0}(R)\right)$ in the notation of $[3, p .516]$.

3. Azumaya algebras. In this section we compute the groups $K_{*} \mathrm{Az}$. The computation was inspired by the calculations of [2] and [14]. I am indebted to C. McGibbon and J. Neisendorfer for suggesting the use of the Comparison Theorem in the proof.

There is a functor End: $\mathbf{F P} \rightarrow \mathbf{A z}$ in $S M C a t$, which sends a faithful projective $R$-module $P$ to its endomorphism ring $\operatorname{End}(P)$, and sends the automorphism $\alpha$ of $P$ to conjugation by $\alpha$. This induces a map End: $\mathrm{Gl}_{\otimes}(R) \rightarrow \operatorname{Aut}(\mathrm{Az})$. The following result is proven in [2] and on page 74 of [3]: 
Proposition 6 (Rosenberg-Zelinsky). There is an exact sequence

$$
1 \rightarrow U(R) \rightarrow \mathrm{Gl}_{\otimes}(R) \stackrel{\text { End }}{\rightarrow} \operatorname{Aut}(\mathrm{Az}) \rightarrow T \operatorname{Pic}(R) \rightarrow 1,
$$

where $T \operatorname{Pic}(R)$ is the torsion subgroup of $\operatorname{Pic}(R)$.

We consider $T \operatorname{Pic}(R)$ to represent outer automorphisms, and would like a category of inner automorphisms. We define In to be the image of End. In is the monoidal subcategory of Az whose objects are the $\operatorname{End}(P)$, and whose arrows are "inner" automorphisms. The group $\operatorname{Aut}(\operatorname{In})=\operatorname{colim} \operatorname{In} \operatorname{Aut}\left(M_{n}(R)\right)$ is the group $\mathrm{PGl}_{\otimes}(R)=\mathrm{Gl}_{\otimes}(R) / U(R)$ (cf. pages 108, 119 of [2] and page 74 of [3]). We thus have short exact sequences of groups $1 \rightarrow U(R) \rightarrow \mathrm{Gl}_{\otimes}(R) \rightarrow \mathrm{PGl}_{\otimes}(R) \rightarrow 1$ and $1 \rightarrow \mathrm{PGl}_{\otimes}(R) \rightarrow \operatorname{Aut}(\mathrm{Az}) \rightarrow T \operatorname{Pic}(R) \rightarrow 1$. The sequence Pic $\rightarrow$ FP $\rightarrow$ In gives rise to a commutative diagram of spaces

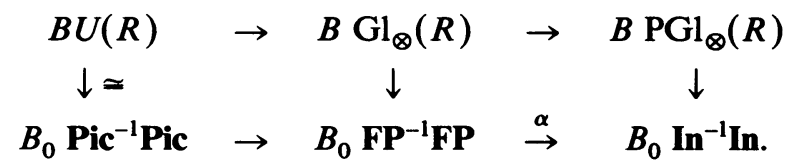

The top row is a fibration, and the bottom row is a sequence of infinite loop spaces and infinite loop maps. The left vertical arrow is a homotopy equivalence of infinite loop spaces by Proposition 2. As the bottom composite is trivial, there is an infinite loop space map from $B_{0}$ Pic $^{-1}$ Pic to the fiber $X$ of the lower right horizontal map $\alpha$. Summarizing, there is a map of fibrations

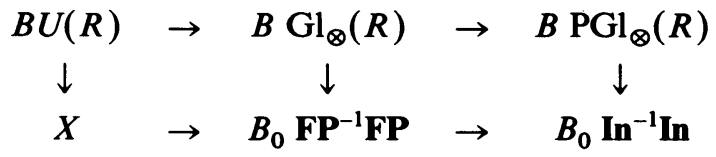

in which the map $B U(R) \rightarrow X$ is an $H$-map. Now $\mathrm{PGl}_{\otimes}(R)$ acts trivially on $H_{*}\left(B U(R)\right.$ ) because $U(R)$ is central in $\mathrm{Gl}_{\otimes}(R)$ (any element of $\mathrm{PGl}_{\otimes}(R)$ induces the identity map on $B U(R))$. Moreover, $\pi_{1}\left(B_{0} \mathbf{I n}^{-1} \mathbf{I n}\right)$ acts trivially on $H_{*}(X)$ because $\alpha$ is an $H$-map and $X$ is connected (see [5, p. 16-09]). Hence the Comparison Theorem [8, p. 355] applies: as the base and total space maps are homology isomorphisms (by Proposition 3), the infinite loop space map $B U(R) \rightarrow X$ is a homology isomorphism, hence a homotopy equivalence. We have proven:

TheOREM 7. $B U(R) \rightarrow B \mathrm{Gl}_{\otimes}(R)^{+} \rightarrow B \mathrm{PGl}_{\otimes}(R)^{+}$is a homotopy fibration.

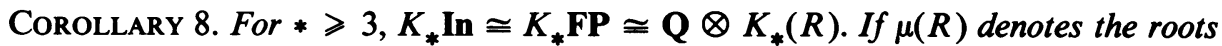
of unity of $R$,

$$
K_{2} \mathbf{I n}=\mu(R) \oplus K_{2} \mathbf{F P}=\mu(R) \oplus\left(\mathbf{Q} \otimes K_{2}(R)\right) .
$$

Finally, $K_{1} \mathbf{I n}=K_{1} \mathbf{F P} / \operatorname{im} U(R)$ and $K_{0} \mathbf{I n}=U^{+}\left(\mathbf{Q} \otimes K_{0}(R)\right) / \operatorname{im}(\operatorname{Pic}(R))$.

Proof. Use the long exact homotopy sequence and the fact that $\pi_{*} B U=0$ for $* \neq 1$, as well as Proposition 5. The only subtleties are that in the sequence $0 \rightarrow K_{2} \mathbf{F P} \rightarrow K_{2}(\mathrm{In}) \rightarrow U(R) \rightarrow K_{1}$ FP the left map splits (by divisibility of $K_{2}$ FP) and that the kernel of the right map is the torsion subgroup $\mu(R)$ of $U(R)$. 
THEOREM 9. There is a long exact sequence in $K$-theory:

$$
\cdots K_{*+1} \mathrm{Az} \rightarrow K_{*} \text { Pic } \rightarrow K_{*} \text { FP } \rightarrow K_{*} \mathrm{Az} \cdots
$$

In particular: for $* \geqslant 3, K_{*} \mathbf{A z}=K_{*} \mathbf{F P}=\mathbf{Q} \otimes K_{*}(R), K_{2} \mathbf{A z}=\mu(R) \oplus K_{2} \mathbf{F P}=$ $\mu(R) \oplus\left(\mathbf{Q} \otimes K_{2}(R)\right)$,

$$
K_{1} \mathrm{Az}=T \operatorname{Pic}(R) \oplus(\mathbf{Q} / \mathbf{Z} \otimes U(R)) \oplus\left(\mathbf{Q} \otimes S K_{1}(R)\right),
$$

and $K_{0} \mathrm{Az}=\operatorname{Br}(R) \oplus U^{+}\left(\mathbf{Q} \otimes K_{0}(R)\right) /$ im $\operatorname{Pic}(R)$, where $\operatorname{Br}(R)$ is the Brauer group of $R$.

Proof. The map $B$ Aut(In) $\rightarrow B$ Aut(Az) is (up to homotopy) a covering space map with fiber the abelian group $T \operatorname{Pic}(R)$. The commutator groups [Aut(In), Aut(In)] and [Aut(Az), Aut(Az)] are isomorphic. Hence we can perform a $T$ Pic-equivariant plus construction on $B$ Aut(In): for every cell we attach, all translates of the cell are also attached. In this way we obtain the model $B$ Aut(In) ${ }^{+} / T$ Pic for $B \operatorname{Aut}(\mathbf{A z})^{+}$, and a fibration $T \operatorname{Pic}(R) \rightarrow B \operatorname{Aut}(I n)^{+} \rightarrow$ $B$ Aut $(\mathbf{A z})^{+}$. This yields $K_{*} \mathbf{A z}$ for $* \geqslant 2$. Bass's analysis of the low-dimensional terms in [2] gives $K_{0}, K_{1}$ and a fibration $B \mathbf{P i c}^{-1} \mathbf{P i c} \rightarrow B \mathbf{F P}^{-1} \mathbf{F P} \rightarrow B \mathrm{Az}^{-1} \mathbf{A z}$.

REMARK. We have shown that the commutator subgroup $E$ of $\operatorname{PGl}_{\otimes}(R)$ is perfect. In fact, it is the subgroup generated by the images of the elementary matrices in the $\mathrm{Gl}_{n}(R)$, so the fact that $E=[E, E]$ may be deduced from the fact that elementary matrices are commutators in $\mathrm{Gl}_{n}, n \geqslant 3$. More interesting is the following consequence of Corollary 8: the torsion subgroup of $\mathrm{H}_{2}(E)$ is isomorphic to the roots of unity in the ring $R$. It would be interesting to find an explicit description of this isomorphism, especially for $R=C$.

ACKNOWLEDGEMENTS. I would like to thank Z. Fiedorowicz for explaining the relation of monoidal categories and infinite loop spaces to me. I would also like to thank C. McGibbon and J. Neisendorfer for suggesting the use of the Comparison Theorem, circumventing a much more complicated approach.

\section{REFERENCES}

1. J. F. Adams, Infinite loop spaces, Ann. of Math. Studies, no. 90, Princeton Univ. Press, Princeton, N. J., 1978.

2. H. Bass, Lectures on topics in algebraic K-theory, Tata Institute of Fundamental Research, Bombay, 1967.

3. , Algebraic K-theory, Benjamin, New York, 1968.

4. __ Unitary algebraic K-theory, Lecture Notes in Math., vol. 343, Springer-Verlag, New York, 1973.

5. H. Cartan, Séminaire H. Cartan 1959/60, exposé 16, École Norm. Sup., Paris, 1961.

6. D. Grayson, Higher algebraic K-theory: II (after D. Quillen), Lecture Notes in Math., vol. 551, Springer-Verlag, New York, 1976.

7. S. Mac Lane, Categories for the working mathematician, Springer-Verlag, New York, 1971.

8. _ Homology, Springer-Verlag, New York, 1967.

9. J. P. May, $E_{\infty}$ spaces, group completions, and permutative categories, New Developments in Topology, (Proc. Sympos. Algebraic Topology; Oxford, 1972), London Math. Soc. Lecture Note Ser., No. 11, Cambridge Univ. Press, London, 1974, pp. 61-93.

10. $\ldots, E_{\infty}$ ring spaces and $E_{\infty}$ ring spectra, Lecture Notes in Math., vol. 657, Springer-Verlag。 New York, 1977. 
11. J. Milnor, Introduction to algebraic K-theory, Ann. of Math. Studies, no. 72, Princeton Univ. Press, Princeton, N. J., 1971.

12. G. Segal, Categories and cohomology theories, Topology 13 (1974), 293-312.

13. R. W. Thomason, Homotopy colimits in the category of small categories, Math. Proc. Cambridge Philos. Soc. 85 (1979), 91-109.

14. C. A. Weibel, $K V$-theory of categories, (preprint 1979).

15. G. Whitehead, Elements of homotopy theory, Springer-Verlag, New York, 1978.

Department of Mathematics, University of Pennsylvania, Philadelphia, Pennsylvania 19104

Current address: Department of Mathematics, Rutgers University, New Brunswick, New Jersey 08903 